\title{
Is sentinel node biopsy necessary in the radiologically negative axilla in breast cancer?
}

\author{
Felix Jozsa ${ }^{1}(1) \cdot$ Muneer Ahmed ${ }^{1} \cdot$ Rose Baker $^{2} \cdot$ Michael Douek $^{3}$
}

Received: 27 March 2019 / Accepted: 25 May 2019 / Published online: 31 May 2019

๑) Springer Science+Business Media, LLC, part of Springer Nature 2019

\begin{abstract}
Purpose The steady move towards axillary conservatism in breast cancer is based on studies demonstrating that axillary node clearance affords no survival benefit in a subset of patients with a positive pre-operative axillary ultrasound (AUS). However, less attention has been paid to AUS-negative patients who receive sentinel node biopsy as standard.

Methods Previously assembled systematic review data was reassessed to evaluate nodal burden amongst patients with breast cancer and a clinically and radiologically negative axilla.

Results Pooled data from four cohort studies reporting pre-operative axillary ultrasound in 5139 patients with breast cancer show it has a negative predictive rate of 0.951 (95\% confidence interval $0.941-0.960$ ).

Conclusions Reconsidering the use of ultrasound in patients with early breast cancer and non-palpable axillae reveals that sentinel node biopsy itself may represent surgical over-treatment in patients with a negative axillary ultrasound. The implications of this on the future of surgical management of the axilla are discussed.
\end{abstract}

Keywords Axillary ultrasound $\cdot$ Axillary node metastasis $\cdot$ Sentinel node biopsy $\cdot$ Axillary node clearance $\cdot$ Axillary conservatism

Over the past 20 years, the surgical approach to the axilla in breast cancer has become less radical; axillary node clearance (ANC) is used selectively, having previously been favoured in all patients for reduction of local disease recurrence [1].

Results from the ACOSOG Z11 trial [2] demonstrated that in T1-2 invasive breast cancer with axillary node metastasis and no palpable lymphadenopathy, there was no survival benefit in ANC following sentinel node biopsy (SNB) if 2 or fewer nodes were involved, prior to breast surgery and radiotherapy. Z11 thus introduced the clinical need to quantify axillary nodal disease; those with low burden being spared ANC and the risks with which it is associated (functional arm impairment, lymphedema) [3].

Felix Jozsa

felixjozsa@gmail.com

1 Division of Cancer Studies, Research Oncology, King's College London, London SE1 9RT, UK

2 Department of Statistics, School of Business, University of Salford, 612, Maxwell Building, Salford M5 4WT, UK

3 Nuffield Department of Surgical Sciences, University of Oxford, Botnar Research Centre, Oxford OX3 7LD, UK

\section{The clinical value of a pre-operative negative axillary ultrasound}

Many studies have focussed on the use of axillary ultrasound (AUS) to identify high nodal burden in the preoperative axilla. By reconsidering this data to observe the ultrasound negative patients, a trend emerges of low nodal burden in the radiologically negative axilla. AUS may, therefore, have an important role to play in axillary assessment within the context of Z11.

In a retrospective cohort of 1538 breast cancer patients (Barco et al. 2016 [4]), 1151 were AUS-negative and, at SNB, 95.7\% had low nodal burden. Boone et al. (2015) [5] and Moorman et al. (2014) [6] reported 96.0\% $(n=434)$ and 95.8\% ( $n=879)$ of AUS-negative patients respectively with low nodal burden at SNB. Further, Veheuvel et al. (2016) [7] reported $93.45 \%$ of low burden in 2749 AUS-negative patients (see Table 1).

A pooled data analysis with random-effects fitted to a beta-binomial model demonstrates ultrasound's negative predictive value as 0.951 (95\% confidence interval 0.941 to 0.951) (see Fig. 1). 
Table 1 Studies reporting proportion of patients with primary breast cancer and a negative axillary ultrasound, with subsequent low nodal burden at sentinel node biopsy

\begin{tabular}{lccc}
\hline Study & $\begin{array}{l}\text { Number of patients with negative } \\
\text { axillary ultrasound }\end{array}$ & $\begin{array}{l}\text { Number of patients with negative axillary ultra- } \\
\text { sound and low nodal burden at SNB }\end{array}$ & $\begin{array}{l}\text { Negative predictive value } \\
\text { (95\% confidence interval) }\end{array}$ \\
\hline Barco et al. (2016) [5] & 1151 & 1101 & $0.957(0.943-0.967)$ \\
Boone et al. (2015) [6] & 434 & 417 & $0.961(0.938-0.977)$ \\
Moorman (2014) [7] & 879 & 842 & $0.958(0.942-0.970)$ \\
Verheuvel (2016) [8] & 2675 & 2514 & $0.940(0.930-0.948)$ \\
Pooled data & 5139 & 4874 & $0.951(0.941-0.960)$ \\
\hline
\end{tabular}

Fig. 1 Forest plot illustrating the data presented in Table 1

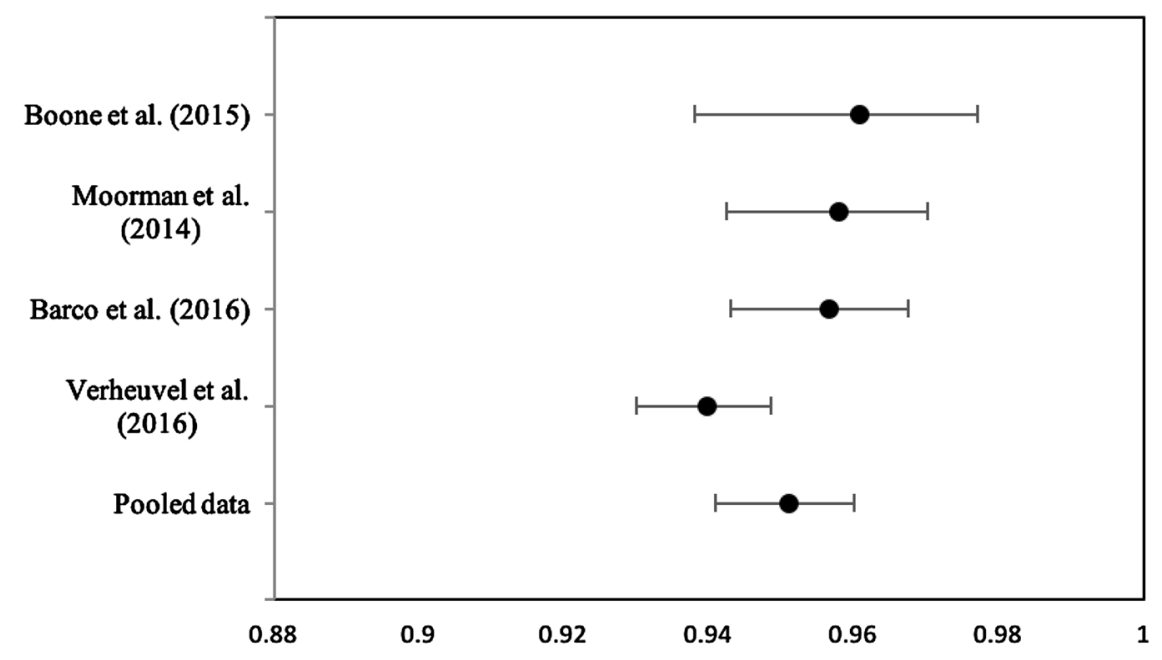

Proportion of patients with negative axillary ultrasound and subsequent low nodal burden with $95 \%$ confidence intervals

\section{Further improving the negative predictive value of preoperative axillary ultrasound}

If radiological information could be combined with salient clinical and histopathological factors to create a risk index, this may enable targeting of the high nodal burden group in those with a radiologically negative axilla (the approximately $4 \%$ of patients, as demonstrated above). Subgroup analysis from ongoing and future trials may elucidate this possibility. One such consideration will be the effect of body mass index (BMI) on axillary assessment—are these patients underdiagnosed at clinical assessment or by AUS? This is an important contemporary point given that rates of obesity have more than doubled worldwide since 1980 [8]. Recent retrospective cohort data shows that nodal burden does not differ amongst obese and non-obese patients clinically diagnosed as node negative [9], however, the ability of ultrasound to stratify burden in the obese cN0 patient subgroup is less clear, and clarification from randomised control trial data will be welcome.

\section{The problem with the ultrasound positive axilla}

These data support a move towards omitting SNB in patients with a clinically and radiologically normal axilla, in the context of early disease with surgery and adjuvant therapy. The adoption of a screening tool demands that all its outcomes be prepared for however, and the clinical simplicity of the ultrasound negative patients is not mirrored in the ultrasound-positive cohort. Here, it has been established that the sensitivity of AUS is not sufficient to be used as a clinical decision-making tool because, when used to bypass SNB following positive AUS, $43.2 \%$ of this group undergo unnecessary ANC procedures owing to false positive results [10]. This picture is further muddied with the use of core needle biopsy (CNB), reportedly having a sensitivity of $88 \%$ (84 to 91) in a recent pooled analysis [11]. Importantly, CNB cannot quantify the nodal burden and is, therefore, not clinically useful in the context of Z11, where mere identification of nodal disease in the axilla is not sufficient; it is necessary 
to stratify low and high nodal burden because the overall survival is only increased with axillary surgical intervention in the latter patient group. Thus, even if one or more suitable lymph node biopsy targets are visualised for CNB, this procedure is not beneficial to the patient as-even if positive - it will not provide the clinician with enough information to decide if further axillary surgery is needed or not. Furthermore, a policy of proceeding to ANC following a positive CNB will inevitably be marred with similarly unacceptably high rates of false positives as seen with AUS in the context of Z11-a sure fate for any axillary assessment technique that is unable to clearly quantify the number of nodes with metastatic disease. For this reason, we recommend the non-palpable, ultrasound-positive axilla be assessed with SNB in the first instance which will allow quantification of the nodal burden before definitive axillary management is decided (Fig. 2).

\section{Anticipated clinical trial results relevant to staging the preoperative axilla in breast cancer}

Various relevant international randomised clinical trials are currently underway (Fig. 2). The SOUND [12] and INSEMA [13] trials randomize patients with a negative AUS to no further axillary intervention or SNB, providing much needed
RCT evidence on this issue. The SENOMAC [14] trial expands upon the Z11 population, including patients undergoing mastectomy. In clinically node-positive patients, the TAXIS [15] trial will look at survival after selective node clearance as a less extensive surgical option to complete ANC.

\section{Conclusions}

Axillary ultrasound provides the clinician with extra information that is not concrete or pathologically conclusive. However, as a cost-effective and non-invasive tool that is widely available, it can preclude the need for axillary surgery in a subgroup of patients with early disease and nonpalpable axillary lymph nodes. Although a positive axillary ultrasound still needs to be followed up with SNB, crucially the overall number of axillary interventions is reduced by the screening and identification of the AUS-negative group, and they are targeted to patients with justified clinical suspicion. This underlines the value of AUS as the first line assessment of the axilla preoperatively. The combined efficacy of AUS with timely and appropriate SNB as outlined in Fig. 2 provides optimal screening of the axilla, and is not improved by addition of less reliable techniques such as CNB that do not provide crucial quantification of the nodal burden, even when positive.
Fig. 2 Flowchart summarising proposed axillary workup for breast cancer patients, with current clinical trials assessing relevant aspects highlighted in red

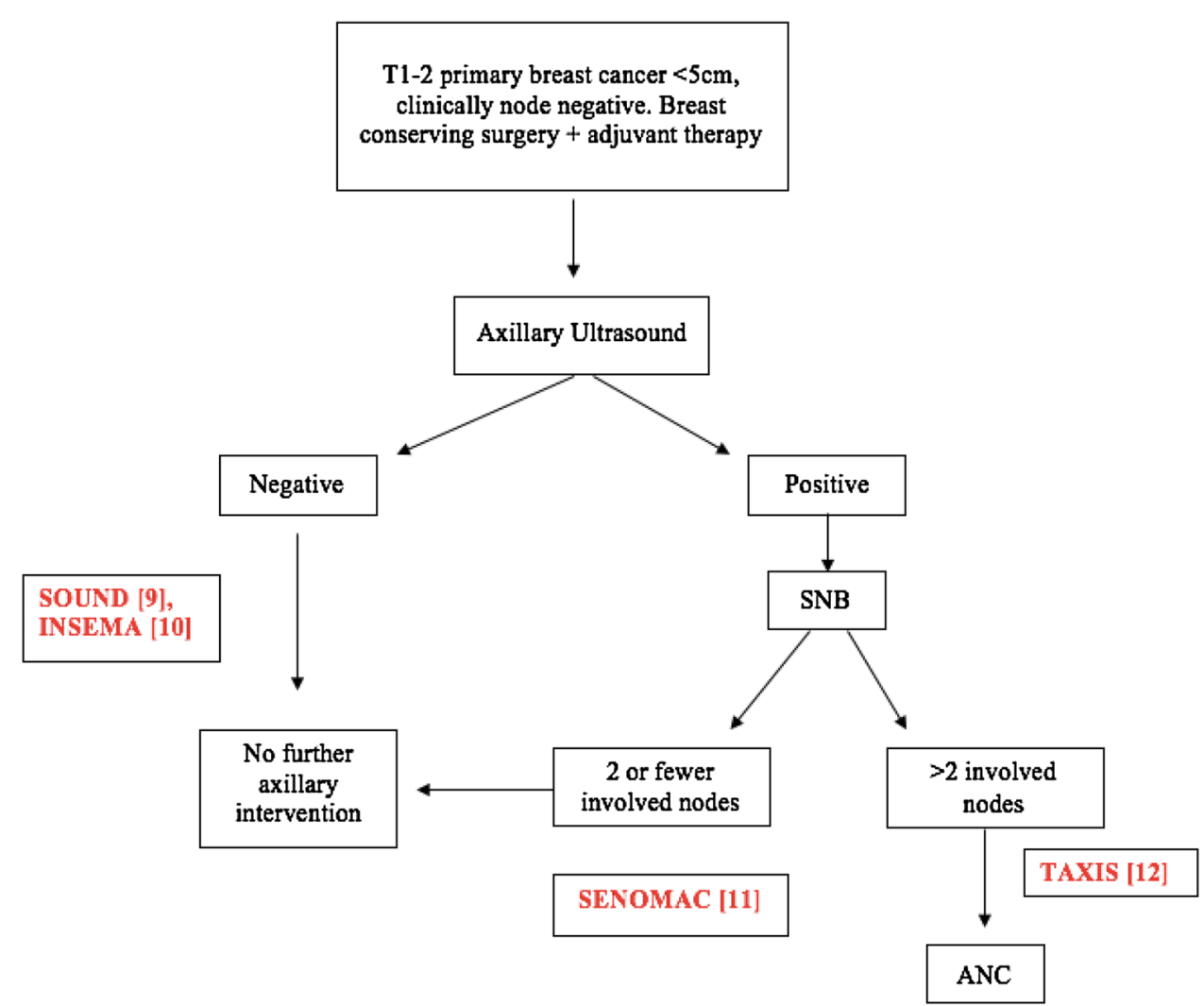


The stark regression in aggressive surgical management of the axilla follows demonstration that no survival benefit is afforded by this approach in select patient groups. Key clinical questions around the point at which intervention should be considered remain, and further trials will aim to clarify these. Our meta-analysis data support the omission of surgical intervention in the clinically and radiologically negative axillae.

\section{Compliance with ethical standards}

Conflict of interest All the authors declare that they have no conflict of interest.

Ethical approval This article does not contain any studies with human participants or animals performed by any of the authors.

\section{References}

1. Jatoi I (2017) Reconsidering axillary surgery for early breast cancer. Indian J Med Res 145(2):155-157

2. Giuliano AE, Hunt KK, Ballman KV et al (2011) Axillary dissection vs no axillary dissection in women with invasive breast cancer and sentinel node metastasis: a randomized clinical trial. JAMA 305(6):569-575

3. Veronesi U, Paganelli G, Viale G, Luini A et al (2003) A randomized comparison of sentinel-node biopsy with routine axillary dissection in breast cancer. N Engl J Med 349:546-553. https:// doi.org/10.1056/nejmoa012782

4. Barco I, Chabrera C, Garcia-Fernandez A, Fraile M, Gonzalez S, Canales L, Lain JM, Gonzalez C, Vidal MC, Vallejo E et al (2017) Role of axillary ultrasound, magnetic resonance imaging, and ultrasound-guided fine-needle aspiration biopsy in the preoperative triage of breast cancer patients. Clin Transl Oncol 19(6):704-710

5. Boone BA, Huynh C, Spangler ML, Sumkin J, Johnson R, McGuire KP, Soran A, Ahrendt GM (2015) Axillary lymph node burden in invasive breast cancer: a comparison of the predictive value of ultrasound-guided needle biopsy and sentinel lymph node biopsy. Clin Breast Cancer 15(5):e243-e248

6. Moorman AM, Bourez RL, Heijmans HJ, Kouwenhoven EA (2014) Axillary ultrasonography in breast cancer patients helps in identifying patients preoperatively with limited disease of the axilla. Ann Surg Oncol 21(9):2904-2910
7. Verheuvel NC, Voogd AC, Tjan-Heijnen VC, Roumen RM (2016) Potential impact of application of Z0011 derived criteria to omit axillary lymph node dissection in node positive breast cancer patients. Eur J Surg Oncol: J Eur Soc Surg Oncol Br Assoc Surg Oncol 42(8):1162-1168

8. Bhurosy T, Jeewon R (2014) Overweight and obesity epidemic in developing countries: a problem with diet, physical activity, or socioeconomic status? Sci World J 2014:964236. https://doi. org/10.1155/2014/964236

9. McCartan D, Stempel M, Eaton A, Morrow M, Pilewskie M (2016) Impact of body mass index on clinical axillary nodal assessment in breast cancer patients. Ann Surg Oncol 23(10):3324-3329. https://doi.org/10.1245/s10434-016-5330-0

10. Ahmed M, Jozsa F, Baker R, Rubio IT, Benson J, Douek M (2017) Meta-analysis of tumour burden in pre-operative axillary ultrasound positive and negative breast cancer patients. Breast Cancer Res Treat 166(2):329-336

11. Balasubramanian I, Fleming CA, Corrigan MA, Redmond HP, Kerin MJ, Lowery AJ (2018) Meta-analysis of the diagnostic accuracy of ultrasound-guided fine-needle aspiration and core needle biopsy in diagnosing axillary lymph node metastasis. Br J Surg 105:1244-1253. https://doi.org/10.1002/bjs.10920

12. Gentilini O, Botteri E, Dadda P, Sangalli C, Boccardo C et al (2016) Physical function of the upper limb after breast cancer surgery. Results from the SOUND (sentinel node vs. observation after axillary Ultra-souND) trial. Eur J Surg Oncol 42(5):685-689. https://doi.org/10.1016/j.ejso.2016.01.020

13. Reimer T, Stachs A, Nekljudova V et al (2017) Restricted axillary staging in clinically and sonographically node-negative early invasive breast cancer (c/iT1-2) in the context of breast conserving therapy: first results following commencement of the intergroupSentinel-Mamma (INSEMA) trial. Geburtshilfe Frauenheilkd 77(2):149-157

14. de Boniface J, Frisell J, Andersson Y et al (2017) Survival and axillary recurrence following sentinel node-positive breast cancer without completion axillary lymph node dissection: the randomized controlled SENOMAC trial. BMC Cancer 17(1):379. https ://doi.org/10.1186/s12885-017-3361-y

15. Henke G, Knauer M, Ribi K et al (2018) Tailored axillary surgery with or without axillary lymph node dissection followed by radiotherapy in patients with clinically node-positive breast cancer (TAXIS) study protocol for a multicenter, randomized phase-III trial. Trials 19(1):667. https://doi.org/10.1186/s13063-018-3021-9

Publisher's Note Springer Nature remains neutral with regard to jurisdictional claims in published maps and institutional affiliations. 